\title{
Asymmetric Mannich Reaction: Synthesis of Novel Chiral 5-(substituted aryl)-1,3,4-Thiadiazole Derivatives with Anti-Plant-Virus Potency
}

https://doi.org/10.1515/hc-2019-0005

Received June 20, 2018; accepted October 26, 2018.

\begin{abstract}
A series of novel chiral 5-(substituted aryl)-1,3,4thiadiazole derivatives was synthesized in an enantioselective three-component Mannich reaction using cinchona alkaloid squaramide catalyst with excellent enantioselectivities (up to $>99 \%$ enantiomeric excess (ee)). The bioassay results showed that these derivatives possessed good to excellent activities against tobacco mosaic virus (TMV).
\end{abstract}

Keywords: anti-plant-virus activity, asymmetric Mannich reaction, chiral 1,3,4-thiadiazole derivatives

\section{Introduction}

The design and synthesis of novel compounds that have value as agricultural therapeutic agents is one of the fundamental objectives of organic chemistry. During the past two decades, the number of non-racemic pesticides on the agrochemical market has been consistently increasing. Many novel non-racemic agrochemicals have desirable biological activity and can be used in smaller quantities because they possess low mammalian toxicity and environmental friendliness [1-3]. For example, metolachlor is a herbicide that was first used as a racemate.

\footnotetext{
* Corresponding author: Song Bai, School of Chemical Engineering, Guizhou Institute of Technology, Guiyang 550003, China; Key Laboratory of Green Pesticide and Agricultural Bioengineering, Ministry of Education, Guizhou University, Guiyang 550025, China; email: Basonmail@163.com

Yunying Zhu, School of Chemical Engineering, Guizhou Institute of Technology, Guiyang 550003, China; Key Laboratory of Green Pesticide and Agricultural Bioengineering, Ministry of Education, Guizhou University, Guiyang 550025, China

Qin Wu, School of Chemical Engineering, Guizhou Institute of Technology, Guiyang 550003, China
}

The $(R)$-enantiomer is inactive, and modern production methods afford only (S)-metolachlor [4].

Due to their proven utility in medicinal chemistry, there has been greater interest in heterocyclic compounds [5-6]. There are a large number of biologically active organic compounds with five-membered rings containing three heteroatoms. The 1,3,4-thiadiazole heterocycle is the most widely existing one. A survey of the available literature shows that the 1,3,4-thiadiazole heterocycle is a key structure associated with many bioactive molecules [7-9]. For example, several biologically active molecules with a 1,3,4-thiadiazole moiety are known to exhibit significant insecticidal [10], antifungal [11], and antiviral activity against TMV properties [12]. However, research on modified 1,3,4-thiadiazole derivatives has mainly focused on racemic ones. Only one work has been reported using 1,3,4-thiadiazole as a substrate in an asymmetric reaction, a study by Hu's group. They gave the corresponding products with moderate yields (37-53\%) and enantioselectivities (43-91\%) [13]. In addition, our group has previously described the asymmetric addition of malonate with benzothiazolyl imine [14], and it was proven that the thiourea-cinchonine alkaloid derivatives were highly efficient catalysts in the synthesis of chiral $\beta$-amino esters bearing a benzothiazole moiety. Based on our technical competence in the field of plant virology, to exploit novel high-efficiency chiral anti-plant-virus agents, we decided to apply the cinchona alkaloid catalyst to synthesis of masked chiral 5-(substituted aryl)-1,3,4-thiadiazole derivatives. Additionally, the antiviral activity of chiral products against TMV from our asymmetric reaction was tested.

\section{Results and Discussion}

For optimization of the experimental conditions, we initially employed commercial quinine and cinchona alkaloid derivatives Q-1-Q-5 (Figure 1) in a one-pot asymmetric catalytic Mannich reaction of

2 open Access. () 2019 Song Bai et al., published by De Gruyter. @๐ $\odot$ This work is licensed under the Creative Commons Attribution alone 4.0 License. 
<smiles>C=CC1CN2CCC1C2C(O)c1ccnc2ccc(OC)cc12</smiles><smiles>C=CC1CN2CCC1C2[C@H](NC(=S)N[Bi])c1ccnc2ccc(O)cc12</smiles>

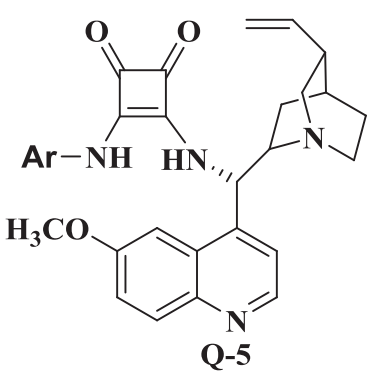

Q-1: $\mathrm{Ar}=3,5-\left(\mathrm{CF}_{3}\right)_{2} \mathrm{C}_{6} \mathrm{H}_{3}$

Q-2: $\mathrm{Ar}=4-\left(\mathrm{OCH}_{3}\right) \mathrm{C}_{6} \mathrm{H}_{4}$ Q-3: $\mathrm{Ar}=4-\left(\mathrm{CF}_{3}\right) \mathrm{C}_{6} \mathrm{H}_{4}$ Q-4: $\mathrm{Ar}=2-(\mathrm{F})-5-\left(\mathrm{CF}_{3}\right) \mathrm{C}_{6} \mathrm{H}_{3}$ Q-5: $A r=3,5-\left(\mathrm{CF}_{3}\right)_{2} \mathrm{C}_{6} \mathrm{H}_{3}$

Figure 1 Structure of commercial quinine and cinchona alkaloid catalysts Q-1-Q-5

Table 1 Optimization of reaction conditions using various catalysts ${ }^{\text {a }}$

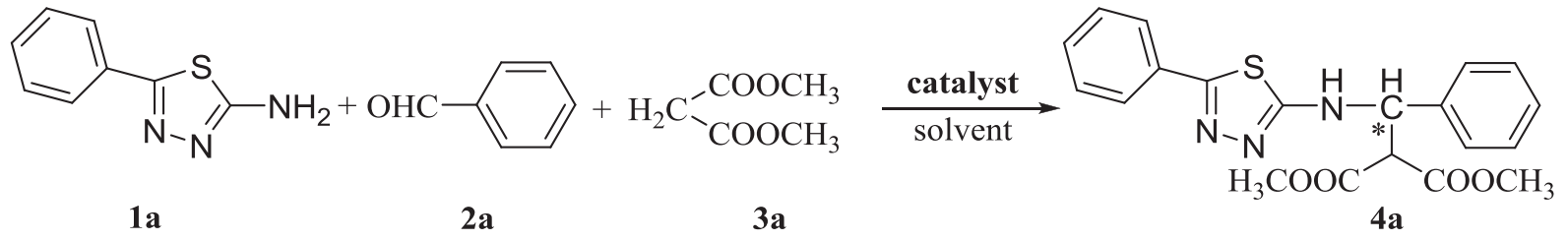

\begin{tabular}{|c|c|c|c|c|c|}
\hline Entry & Catalyst (mol\%) & Temp. $\left({ }^{\circ} \mathrm{C}\right)$ & Solvent & Yield $^{\mathrm{b}}(\%)$ & $\mathrm{ee}^{c}(\%)$ \\
\hline 1 & Quinine (10 mol\%) & 30 & $\mathrm{PhMe}$ & 30 & 58 \\
\hline 2 & Q-1 (10 mol\%) & 30 & PhMe & 40 & 78 \\
\hline 3 & Q-2 (10 mol\%) & 30 & PhMe & 35 & 63 \\
\hline 4 & Q-3 (10 mol\%) & 30 & $\mathrm{PhMe}$ & 43 & 72 \\
\hline 5 & Q-4 (10 mol\%) & 30 & $\mathrm{PhMe}$ & 40 & 70 \\
\hline 6 & Q-5 (10 mol\%) & 30 & $\mathrm{PhMe}$ & 48 & 83 \\
\hline 7 & Q-5 (10 mol\%) & 30 & Tetrahydrofuran & 30 & 71 \\
\hline 8 & Q-5 (10 mol\%) & 30 & $\mathrm{CH}_{2} \mathrm{Cl}_{2}$ & 40 & 78 \\
\hline 9 & Q-5 (10 mol\%) & 30 & Acetone & 35 & 77 \\
\hline 10 & Q-5 (10 mol\%) & 40 & PhMe & 53 & 86 \\
\hline 11 & Q-5 (10 mol\%) & 50 & $\mathrm{PhMe}$ & 61 & 91 \\
\hline 12 & Q-5 (10 mol\%) & 60 & $\mathrm{PhMe}$ & 84 & 93 \\
\hline 13 & Q-5 (10 mol\%) & 70 & $\mathrm{PhMe}$ & 86 & 75 \\
\hline 14 & Q-5 (5 mol\%) & 60 & $\mathrm{PhMe}$ & 65 & 67 \\
\hline 15 & Q-5 (20 mol\%) & 60 & PhMe & 85 & 94 \\
\hline
\end{tabular}

${ }^{a}$ Reactions were carried out with $1.0 \mathrm{mmol}$ of $1,1.0 \mathrm{mmol}$ of $2 \mathrm{a}$, and $1.2 \mathrm{mmol}$ of $3 \mathrm{a}$ in $2.0 \mathrm{~mL}$ of solvent for $60 \mathrm{~h}$.

${ }^{\mathrm{b}}$ Isolated yield.

' Determined by HPLC (Chiralpak IA).

5-phenyl-1,3,4-thiadiazol-2-amine, benzaldehyde, and dimethyl malonate (entry 1-6, Table 1). Quinine was a poor catalyst, whereas cinchona alkaloid-squaramide catalyst Q-5 afforded an especially promising result at $30^{\circ} \mathrm{C}$ for $60 \mathrm{~h}$ (entry 6, Table 1). Next, the influence of solvents on the model reaction was studied using the optimal catalyst Q-5 (entries 6-9, Table 1). Toluene showed higher enantioselectivity than alternative solvents. Four gradients (40 ${ }^{\circ} \mathrm{C}, 50^{\circ} \mathrm{C}, 60^{\circ} \mathrm{C}$, and $70^{\circ} \mathrm{C}$ ) of reaction temperature were evaluated in this reaction (entries 10-13, Table 1). It was determined that $60{ }^{\circ} \mathrm{C}$ was suitable for obtaining high yield and excellent enantioselectivity (entry 12, Table 1). The best results were achieved at $60^{\circ} \mathrm{C}$ with $10 \mathrm{~mol} \%$ catalyst loading in toluene.
Having established the optimal reaction conditions, the substrate generality of this asymmetric Mannich reaction was investigated, and the results are summarized in Table 2. In all cases, the Mannich reaction proceed smoothly when catalyst Q-5 was used, giving the desired chiral 5-(substituted aryl)-1,3,4-thiadiazole derivatives in high isolated yields (71-90\%) and excellent enantiomeric excess (up to $99 \%$ ee). Significantly, both dimethyl malonate (entries 1-3, Table 2) and diethyl malonate (entries 4-8, Table 2) proved to be good substrates with a high yield and ee values. It should be noted that the different configurations were observable using $\mathbf{Q}-\mathbf{5}$ as the catalyst.

The anti-TMV activity of the synthesized chiral compounds was tested using previously reported method [15]. 
Table 2 Substrate scope of the enantioselective Mannich reaction.

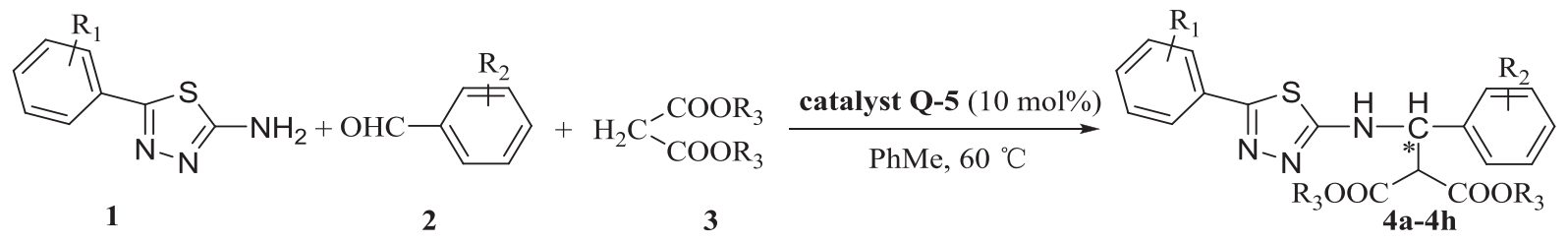

\begin{tabular}{|c|c|c|c|c|c|c|c|}
\hline Entry & 4 & $R_{1}$ & $\mathbf{R}_{2}$ & $\mathbf{R}_{3}$ & Time (h) & Yield $^{\mathrm{b}}(\%)$ & $\mathrm{ee}^{c}(\%)$ \\
\hline 1 & $4 a$ & $\mathrm{H}$ & $\mathrm{H}$ & $\mathrm{Me}$ & 60 & 84 & 93 \\
\hline 2 & $4 b$ & 2, 4-di-Cl & 3, 4-di-Cl & $\mathrm{Me}$ & 60 & 85 & 98 \\
\hline 3 & $4 c$ & $3-F$ & 3, 4-di-Cl & $\mathrm{Me}$ & 60 & 81 & 87 \\
\hline 4 & $4 d$ & $2,4-\mathrm{di}-\mathrm{Cl}$ & 2, 3-di-Cl & Et & 60 & 80 & 95 \\
\hline 5 & $4 e$ & 2, 4-di-Cl & $2-F$ & Et & 72 & 75 & 80 \\
\hline 6 & $4 f$ & $4-\mathrm{Cl}$ & 2, 3-di-Cl & Et & 60 & 90 & $>99$ \\
\hline 7 & $4 g$ & $4-\mathrm{Cl}$ & $2-F$ & Et & 60 & 82 & 90 \\
\hline 8 & $4 h$ & $3-F$ & $3,4-\mathrm{di}-\mathrm{Cl}$ & Et & 72 & 71 & 84 \\
\hline
\end{tabular}

a Reactions were carried out with $1.0 \mathrm{mmol}$ of $1,1.0 \mathrm{mmol}$ of $2 \mathrm{a}, 1.2 \mathrm{mmol}$ of $3 \mathrm{a}$ and $10 \mathrm{~mol} \% \mathrm{Q}-5 \mathrm{in} 2.0 \mathrm{~mL}$ of toluene.

${ }^{\mathrm{b}}$ Isolated yield.

' Determined by HPLC (Chiralpak IA).

Table $3 \mathrm{EC}_{50}$ values of target compounds against TMV

\begin{tabular}{lcc}
\hline Compound & \multicolumn{2}{c}{ EC $_{50}$ for anti-TMV } \\
\cline { 2 - 3 } & $\begin{array}{c}\text { Curative activity } \\
(\mu \mathrm{g} / \mathrm{mL})\end{array}$ & $\begin{array}{c}\text { Protective activity } \\
(\mu \mathrm{g} / \mathrm{mL})\end{array}$ \\
\hline 4b & $803.4 \pm 3.7$ & $361.5 \pm 4.2$ \\
4c & $781.2 \pm 2.6$ & $402.4 \pm 2.5$ \\
4d & $823.7 \pm 2.1$ & $705.6 \pm 3.2$ \\
4e & $813.4 \pm 3.5$ & $380.2 \pm 24$ \\
4f & $605.1 \pm 1.8$ & $264.2 \pm 2.0$ \\
4g & $465.6 \pm 1.9$ & $342.3 \pm 2.9$ \\
4h & $327.3 \pm 3.2$ & $254.8 \pm 4.1$ \\
Ribavirin $^{b}$ & $850.0 \pm 3.1$ & $819.5 \pm 2.4$ \\
\hline
\end{tabular}

a Average of three replicates.

${ }^{\mathrm{b}}$ The commercial product ribavirin served as the control.

${ }^{c}$ Curative activity refers to the therapeutic efficacy of drugs on the plant that have infected with TMV virus.

d Protective activity refers to the ability of drugs to protect plants treated with those drugs from TMV viral infection.

As shown in Table 3. Most chiral compounds exhibited moderate to good antiviral activity against TMV in vivo, and some of them even exhibited higher antiviral activity than the commercially available plant antiviral agent ribavirin.

\section{Conclusions}

We developed an efficient one-pot three-component selective catalytic method for the synthesis of masked chiral 5-(substituted aryl)-1,3,4-thiadiazole derivatives using chiral cinchona alkaloid-squaramide catalyst in good yields with excellent enantioselectivities (up to $99 \%$ ee).
Several new chiral derivatives from our reaction showed potential anti-plant virus activities against TMV.

\section{Experimental}

IR spectra were recorded on a Bruker Vector 22 spectrometer. NMR spectra were recorded at $500 \mathrm{MHz}\left({ }^{1} \mathrm{H}\right)$ and $125 \mathrm{MHz}\left({ }^{13} \mathrm{H}\right)$ on a JEOL-ECX 500 NMR spectrometer using TMS as an internal standard and DMSO- $\mathrm{d}_{6}$ or $\mathrm{CDCl}_{3}$ as a solvent. Mass spectral studies were conducted on an Agilent 5973 organic mass spectrometer. Elemental analysis was performed on an Elementar Vario-III CHN analyzer. High-performance liquid chromatography (HPLC) analysis was performed on an Agilent 1100/1200 Series system using a Daicel Chiralcel IA Column $(250 \mathrm{~mm} \times$ $4.6 \mathrm{~mm})$. HPLC analysis: Hexane/IPA $=70 / 30,1.0 \mathrm{~mL} \cdot \mathrm{min}^{-}$ ${ }^{1}$. Analytical TLC was performed on silica gel GF254. Except where otherwise stated, all reagents were purchased from Aladdin-reagent Inc. and Sigma-Aldrich Inc., and all the commercial reagents were used as received.

General experimental procedure to prepare 4a-4h: Diethyl malonate or dimethyl malonate $(1.20 \mathrm{mmol})$ in toluene $(2.0 \mathrm{~mL})$ was added all at once to a mixture of 5-(substituted aryl)-1,3,4-thiadiazole-2-amine $(1.0 \mathrm{mmol})$, aldehyde $(1.0 \mathrm{mmol})$, and chiral catalyst Q-5 $(10 \mathrm{~mol} \%)$ at $60{ }^{\circ} \mathrm{C}$ and stirred for $60-72 \mathrm{~h}$. After completion of the reaction, the reaction mixture was directly purified by preparative TLC hexane/ethyl ether $=3 / 1$ afforded the desired compounds 4a-4h. 
(+)-Dimethyl 2-(phenyl((5-phenyl-1,3,4-thiadiazol2-yl)amino)methyl)malonate (+)-4a, yield $84 \%$; white solid; $93 \%$ ee as determined by HPLC $[\lambda=210 \mathrm{~nm}, \operatorname{tr}$ (major) $=9.07 \mathrm{~min}, \operatorname{tr}($ minor $)=9.86 \mathrm{~min}] ;[\alpha]_{\mathrm{D}}{ }^{25}=+76.2(\mathrm{c}=0.95$, $\mathrm{CHCl}_{3}$ ); mp 128-129 ${ }^{\circ} \mathrm{C}$; IR $\left(\mathrm{KBr}, \mathrm{cm}^{-1}\right)$ v:3311, 3080, 2953, 2920, 1620, 1558, 1506, 1458, 1375, 1259, 1072, 975, $758 \mathrm{~cm}^{-1}$; ${ }^{1} \mathrm{H}$ NMR (DMSO-d $\left.\mathrm{d}_{6}\right): \delta(\mathrm{ppm}) 7.74-7.72(\mathrm{~m}, 2 \mathrm{H}), 7.46-7.28$ (m, $8 \mathrm{H}), 5.45-5.41(\mathrm{~m}, 1 \mathrm{H}), 4.14-4.12(\mathrm{~m}, 1 \mathrm{H}), 3.68(\mathrm{~s}, 3 \mathrm{H}), 3.45(\mathrm{~s}$, 3H); ${ }^{13}$ C NMR (DMSO-d $): \delta$ (ppm) 167.9, 167.4, 166.8, 157.4, 139.2, 130.4, 130.3, 129.6, 129.0, 128.9, 127.9, 126.9, 58.6, 57.7, 56.2, 53.3, 52.0; MS(ESI): $\mathrm{m} / \mathrm{z}=398\left([\mathrm{M}+\mathrm{H}]^{+}\right), 420\left([\mathrm{M}+\mathrm{Na}]^{+}\right)$, 436 $\left([\mathrm{M}+\mathrm{K}]^{+}\right)$. Anal. Calcd for $\mathrm{C}_{20} \mathrm{H}_{19} \mathrm{~N}_{3} \mathrm{O}_{4} \mathrm{~S}: \mathrm{C}, 60.44 ; \mathrm{H}, 4.82$; N, 10.57; Found: C, 60.58; H, 4.95; N, 10.46.

(-)-Dimethyl 2-((3,4-dichlorophenyl)((5-(2,4-dichl orophenyl)-1,3,4-thiadiazol-2-yl)amino)methyl) malonate (-)-4b, yield $85 \%$; white solid; $98 \%$ ee as determined by HPLC $[\lambda=210 \mathrm{~nm}, \operatorname{tr}$ (major) $=7.16 \mathrm{~min}, \operatorname{tr}($ minor $)=7.73$ $\min ] ;[\alpha]_{D}^{25}=-81.6\left(\mathrm{c}=0.95, \mathrm{CHCl}_{3}\right) ; \mathrm{mp} 177-179{ }^{\circ} \mathrm{C}$; IR $(\mathrm{KBr}$, $\left.\mathrm{cm}^{-1}\right) v: 3201,3003,2951,2916,1661,1550,1435,1280,1232$, 1168, 1062, 979, $823 \mathrm{~cm}^{-1} ;{ }^{1} \mathrm{H}$ NMR $\left(\mathrm{CDCl}_{3}\right): \delta(\mathrm{ppm}) 8.13(\mathrm{~d}$, $J=8.6 \mathrm{~Hz}, 1 \mathrm{H}), 7.51(\mathrm{~d}, J=1.7 \mathrm{~Hz}, 1 \mathrm{H}), 7.47(\mathrm{~d}, J=1.75 \mathrm{~Hz}$, $1 \mathrm{H}), 7.42-7.31(\mathrm{~m}, 2 \mathrm{H}), 7.27-7.25(\mathrm{~m}, 1 \mathrm{H}), 5.63(\mathrm{~d}, J=4.5 \mathrm{~Hz}$, $1 \mathrm{H}), 3.96(\mathrm{~d}, J=4.6 \mathrm{~Hz}, 1 \mathrm{H}), 3.76(\mathrm{~s}, 3 \mathrm{H}), 3.72(\mathrm{~s}, 3 \mathrm{H}) ;{ }^{13} \mathrm{C} \mathrm{NMR}$ $\left(\mathrm{CDCl}_{3}\right): \delta(\mathrm{ppm}) 169.3,168.3,166.8,153.5,138.5,136.2,133.2$, 132.6, 132.0, 131.3, 130.9, 130.1, 128.8, 128.1, 127.7, 126.1, 57.9, 56.4, 53.4, 53.1; MS(ESI): $\mathrm{m} / \mathrm{z}=536\left([\mathrm{M}+\mathrm{H}]^{+}\right), 558\left([\mathrm{M}+\mathrm{Na}]^{+}\right)$. Anal. Calcd for $\mathrm{C}_{20} \mathrm{H}_{15} \mathrm{Cl}_{4} \mathrm{~N}_{3} \mathrm{O}_{4} \mathrm{~S}$ : C, 44.88; H, 2.82; N, 7.85; Found: C, 44.79; H, 2.95; N, 7.90.

(+)-Dimethyl 2-((3,4-dichlorophenyl)((5-(3-fluoro phenyl)-1,3,4-thiadiazol-2-yl)amino) methyl) malonate (+)-4c, yield $81 \%$; white solid; $87.7 \%$ ee as determined by HPLC $[\lambda=270 \mathrm{~nm}$, tr (major) $=14.36 \mathrm{~min}$, tr $($ minor $)=10.17 \mathrm{~min}] ;[\alpha]_{\mathrm{D}}{ }^{25}=+79.3\left(\mathrm{c}=0.81, \mathrm{CHCl}_{3}\right) ; \mathrm{mp}$ 151-152 ${ }^{\circ} \mathrm{C}$; IR $\left(\mathrm{KBr}, \mathrm{cm}^{-1}\right)$ v: 3385, 3053, 2953, 2918, 1622, 1583, 1506, 1471, 1394, 1240, 1197, 980, 783; ' $\mathrm{H}$ NMR (DMSO$\left.\mathrm{d}_{6}\right): \delta(\mathrm{ppm}) 8.77(\mathrm{~d}, J=9.1 \mathrm{~Hz}, 1 \mathrm{H}), 7.61(\mathrm{~d}, J=8.5 \mathrm{~Hz}, 1 \mathrm{H})$, $7.54-7.39(\mathrm{~m}, 5 \mathrm{H}), 7.27(\mathrm{t}, J=8.0 \mathrm{~Hz}), 5.47(\mathrm{t}, J=9.2 \mathrm{~Hz}, 1 \mathrm{H})$, $4.22(\mathrm{~d}, J=9.7 \mathrm{~Hz}, 1 \mathrm{H}), 3.64$ (s, 3H), 3.49 (s, 3H); ${ }^{13} \mathrm{C}$ NMR (DMSO-d $\left.\mathrm{d}_{6}\right): \delta$ (ppm) 167.9, 167.3, 166.8, 163.7, 161.8, 156.7, 140.5, 133.0, 131.9, 131.5, 131.1, 129.9, 128.7, 123.3, 117.3, 113.4, 57.1, 56.9, 53.3, 53.2; ${ }^{19}$ FNMR (200 MHz, DMSO-d 6 ) $\delta:-112.0$; MS (ESI): $\mathrm{m} / \mathrm{z}=484\left([\mathrm{M}+\mathrm{H}]^{+}\right), 506\left([\mathrm{M}+\mathrm{Na}]^{+}\right), 522\left([\mathrm{M}+\mathrm{K}]^{+}\right)$. Anal. Calcd for $\mathrm{C}_{20} \mathrm{H}_{16} \mathrm{Cl}_{2} \mathrm{FN}_{3} \mathrm{O}_{4} \mathrm{~S}: \mathrm{C}, 49.60 ; \mathrm{H}, 3.33 ; \mathrm{N}, 8.68$; Found: C, 49.45; H, 3.31; N, 8.64.

(-)-Diethyl2-((2,3-dichlorophenyl)((5-(2,4-dichloro phenyl)-1,3,4-thiadiazol-2-yl)amino)methyl) malonate (-)-4d, yield $80 \%$; white solid; $95 \%$ ee as determined by HPLC $[\lambda=210 \mathrm{~nm}, \operatorname{tr}$ (major) $=12.48 \mathrm{~min}, \operatorname{tr}($ minor $)=$ $13.71 \mathrm{~min}] ;[\alpha]_{\mathrm{D}}^{25}=-78.6\left(\mathrm{c}=0.94, \mathrm{CHCl}_{3}\right) ; \mathrm{mp} 156-158^{\circ} \mathrm{C}$; IR $\left(\mathrm{KBr}, \mathrm{cm}^{-1}\right) v: 3225,3076,2954,2916,1679,1587,1496,1375$, 1244, 1160, 1035, 980, 833; ${ }^{1} \mathrm{H}$ NMR $\left(\mathrm{CDCl}_{3}\right): \delta(\mathrm{ppm}) 8.12(\mathrm{~d}$,
$J=8.6 \mathrm{~Hz}, 1 \mathrm{H}), 7.46(\mathrm{~d}, J=1.7 \mathrm{~Hz}, 1 \mathrm{H}), 7.43(\mathrm{~d}, J=8.0 \mathrm{~Hz}$, 1H),7.39-7.36 (m, 2H), 7.31 (dd, $J=2.3,2.2 \mathrm{~Hz}, 1 \mathrm{H}), 7.20-7.16$ (t, $J=8.0 \mathrm{~Hz}, 1 \mathrm{H}), 5.97(\mathrm{dd}, J=4.0,4.0 \mathrm{~Hz}, 1 \mathrm{H}), 4.28-4.13$ (m, 5H), 1.26-1.16 (m, 6H); ${ }^{13} \mathrm{C}$ NMR $\left(\mathrm{CDCl}_{3}\right): \delta(\mathrm{ppm}) 169.4$, 168.3, 166.6, 153.4, 137.7, 136.1, 133.8, 132.0, 131.2, 130.4, 130.1, 128.2, 127.7, 127.6, 126.3, 62.6, 62.2, 57.1, 53.7, 14.1, 13.9; MS(ESI): $m / z=564\left([\mathrm{M}+\mathrm{H}]^{+}\right), 586\left([\mathrm{M}+\mathrm{Na}]^{+}\right), 602\left([\mathrm{M}+\mathrm{K}]^{+}\right)$. Anal. Calcd for $\mathrm{C}_{22} \mathrm{H}_{19} \mathrm{Cl}_{4} \mathrm{~N}_{3} \mathrm{O}_{4} \mathrm{~S}$ : C, 46.91; $\mathrm{H}, 3.40$; N, 7.46; Found: C, 46.87; H, 3.33; N, 7.56.

(+)-Diethyl 2-(((5-(2,4-dichlorophenyl)-1,3,4-thiadiazol-2-yl)amino)(2-fluorophenyl) methyl) malonate (+)-4e, yield $75 \%$; white solid; $80.0 \%$ ee as determined by HPLC $[\lambda=210 \mathrm{~nm}, \operatorname{tr}$ (major) $=6.17 \mathrm{~min}, \operatorname{tr}($ minor $)=6.83$ $\min ] ;[\alpha]_{D}{ }^{25}=+81.2\left(\mathrm{c}=0.66, \mathrm{CHCl}_{3}\right) ; \mathrm{mp} 147-149^{\circ} \mathrm{C}$; IR $(\mathrm{KBr}$, $\left.\mathrm{cm}^{-1}\right) v: 3361,3228,2953,2916,1663,1548,1456,1292,1236$, 1166, 1037, 979, 802; ${ }^{1} \mathrm{H}$ NMR (DMSO-d $)$ ) $\delta(\mathrm{ppm}) 8.85$ (d, $J=8.6 \mathrm{~Hz}, 1 \mathrm{H}), 8.00(\mathrm{~d}, J=8.6 \mathrm{~Hz}, 1 \mathrm{H}), 7.80(\mathrm{~S}, 1 \mathrm{H}), 7.55(\mathrm{~d}$, $J=7.4 \mathrm{~Hz}, 2 \mathrm{H}), 7.35(\mathrm{~s}, 1 \mathrm{H}), 7.22-7.19(\mathrm{~m}, 2 \mathrm{H}), 5.78(\mathrm{t}, J=9.7$ $\mathrm{Hz}, 1 \mathrm{H}), 4.16-4.13(\mathrm{~m}, 3 \mathrm{H}), 3.92-3.91(\mathrm{~m}, 2 \mathrm{H}), 1.13-1.12(\mathrm{~m}$, 3H), 0.91-0.90 (m, 3H); ${ }^{13} \mathrm{C}$ NMR (DMSO-d ${ }_{6}$ ): $\delta$ (ppm) 169.3, 166.8, 166.1, 161.5, 151.9, 135.4, 131.9, 131.8, 130.7, 130.4, 130.0, 128.7, 128.5, 126.1, 125.0, 115.8, 62.0, 61.8, 56.9, 52.3, 14.3, 13.9; ${ }^{19} \mathrm{FNMR}(200 \mathrm{MHz}$, DMSO-d 6 ) $\delta$ : -116.8; MS(ESI): $\mathrm{m} / \mathrm{z}=512\left([\mathrm{M}+\mathrm{H}]^{+}\right), 534\left([\mathrm{M}+\mathrm{Na}]^{+}\right), 550\left([\mathrm{M}+\mathrm{K}]^{+}\right)$. Anal. Calcd for $\mathrm{C}_{22} \mathrm{H}_{20} \mathrm{Cl}_{2} \mathrm{FN}_{3} \mathrm{O}_{4} \mathrm{~S}$ : C, 51.57; H, 3.93; N, 8.20; Found: C, 51.43; H, 3.79; N, 8.08.

(-)-Diethyl 2-((5-(4-chlorophenyl)-1,3,4-thiadiazol-2-yl)amino)(2,3-dichlorophenyl) methyl) malonate (-)-4f, yield 90\%; white solid; $>99 \%$ ee as determined by HPLC $[\lambda=210 \mathrm{~nm}$, tr (major) $=22.78 \mathrm{~min}, \operatorname{tr}$ (minor) $=$ $26.87 \mathrm{~min}] ;[\alpha]_{\mathrm{D}}^{25}=-63.8\left(\mathrm{c}=0.11, \mathrm{CHCl}_{3}\right) ; \mathrm{mp} 142-144^{\circ} \mathrm{C}$; IR $\left(\mathrm{KBr}, \mathrm{cm}^{-1}\right) v: 3421,3226,2976,2918,1653,1521,1477,1273$, $1226,1159,1033,981,829 ;{ }^{1} \mathrm{H}$ NMR $\left(\mathrm{CDCl}_{3}\right): \delta(\mathrm{ppm}) 7.70(\mathrm{~d}$, $J=8.0 \mathrm{~Hz}, 2 \mathrm{H}), 7.44-7.36(\mathrm{~m}, 3 \mathrm{H}), 7.21(\mathrm{t}, J=8.0 \mathrm{~Hz}, 1 \mathrm{H})$, $5.87(\mathrm{~s}, 1 \mathrm{H}), 4.25-4.11(\mathrm{~m}, 5 \mathrm{H}), 1.26(\mathrm{t}, J=8.0 \mathrm{~Hz}, 3 \mathrm{H}), 1.19$ $(\mathrm{t}, J=6.9 \mathrm{~Hz}, 3 \mathrm{H}) ;{ }^{13} \mathrm{C} \mathrm{NMR}\left(\mathrm{CDCl}_{3}\right): \delta(\mathrm{ppm}) 168.1,168.0$, 166.5, 157.7, 137.5, 136.1, 133.8, 131.2, 130.5, 129.2, 129.1, 128.1, 127.7, 126.3, 62.6, 62.2, 57.6, 53.8, 14.1, 13.9; MS (ESI): $\mathrm{m} / \mathrm{z}=$ $530\left([\mathrm{M}+\mathrm{H}]^{+}\right), 552\left([\mathrm{M}+\mathrm{Na}]^{+}\right)$. Anal. Calcd for $\mathrm{C}_{22} \mathrm{H}_{20} \mathrm{Cl}_{3} \mathrm{~N}_{3} \mathrm{O}_{4} \mathrm{~S}$ : C, 49.97; H, 3.81; N, 7.95; Found: C, 49.67; H, 3.61; N, 7.55.

(+)-Diethyl 2-(((5-(4-chlorophenyl)-1,3,4-thiadiazol-2-yl)amino)(2-fluorophenyl)methyl) malonate (+)-4g, yield $82 \%$; white solid; $90 \%$ ee as determined by HPLC $[\lambda=210 \mathrm{~nm}, \operatorname{tr}$ (major) $=7.37 \mathrm{~min}, \operatorname{tr}($ minor $)=$ $6.80 \mathrm{~min}] ;[\alpha]_{\mathrm{D}}^{25}=+99.1\left(\mathrm{c}=1.15, \mathrm{CHCl}_{3}\right) ; \mathrm{mp} 163-165^{\circ} \mathrm{C}$; IR $\left(\mathrm{KBr}, \mathrm{cm}^{-1}\right) v 3444,3365,3209,2918,1653,1521,1489,1290$, 1232, 1136, 1035, 981, $831 \mathrm{~cm}^{-1}$; ${ }^{1} \mathrm{H}$ NMR (DMSO-d $)$ ): $\delta$ (ppm) $7.76(\mathrm{~d}, J=8.6 \mathrm{~Hz}, 2 \mathrm{H}), 7.53-7.51$ (m, 3H), 7.35-7.34 (m, 1H), 7.22-7.17 (m, 2H), 5.74-5.70 (s, 1H), 4.14-3.91 (m, 5H), 1.28 $(\mathrm{t}, J=6.8 \mathrm{~Hz}, 3 \mathrm{H}), 0.91(\mathrm{t}, J=6.9 \mathrm{~Hz}, 3 \mathrm{H}) ;{ }^{13} \mathrm{C}$ NMR (DMSO$\left.\mathrm{d}_{6}\right): \delta(\mathrm{ppm}) 167.8,166.8,166.1,156.3,134.8,129.9,129.7$, 
128.5, 126.2, 125.0, 120.0, 62.0, 61.8, 56.2, 14.3, 14.0; ${ }^{19} \mathrm{FNMR}$ $\left(200 \mathrm{MHz}, \mathrm{DMSO}_{-} \mathrm{d}_{6}\right) \delta:-116.8 ; \mathrm{MS}(\mathrm{ESI}): \mathrm{m} / \mathrm{z}=478\left([\mathrm{M}+\mathrm{H}]^{+}\right)$, $500\left([\mathrm{M}+\mathrm{Na}]^{+}\right), 516\left([\mathrm{M}+\mathrm{K}]^{+}\right)$. Anal. Calcd for $\mathrm{C}_{22} \mathrm{H}_{21} \mathrm{ClFN}_{3} \mathrm{O}_{4} \mathrm{~S}$ : C, 55.29; H, 4.43; N, 8.79; Found: C, 55.40; H, 4.57; N, 8.67.

(+)-Diethyl 2-((3,4-dichlorophenyl)((5-(3-fluoro phenyl)-1,3,4-thiadiazol-2-yl)amino) methyl) malonate (+)-4h, yield $71 \%$; White solid; $84.6 \%$ ee as determined by HPLC $[\lambda=210 \mathrm{~nm}$, tr (major) $=16.62 \mathrm{~min}$, tr $($ minor $)=11.44 \mathrm{~min}] ;[\alpha]_{\mathrm{D}}{ }^{25}=+131.2\left(\mathrm{c}=0.69, \mathrm{CHCl}_{3}\right) ; \mathrm{mp}$ 138-140 ${ }^{\circ} \mathrm{C}$; IR $\left(\mathrm{KBr}, \mathrm{cm}^{-1}\right)$ v: 3442, 3040, 2958, 2920, 1620, 1540, 1506, 1471, 1380, 1260, 1228, 1031, 779; ${ }^{1} \mathrm{H}$ NMR (DMSO-d $): \delta(\mathrm{ppm}) 8.82(\mathrm{~d}, J=8.6 \mathrm{~Hz}, 1 \mathrm{H}), 7.73(\mathrm{~d}, J=1.7$ $\mathrm{Hz}, 1 \mathrm{H}), 7.65(\mathrm{~d}, J=8.0 \mathrm{~Hz}, 1 \mathrm{H}), 7.59-7.45(\mathrm{~m}, 4 \mathrm{H}), 7.32$ (t, $J=$ $8.0 \mathrm{~Hz}, 1 \mathrm{H}), 5.51$ (t, $J=9.15 \mathrm{~Hz}, 1 \mathrm{H}), 4.20-3.98$ (m, 5H), 1.13 (t, $J=6.8 \mathrm{~Hz}, 3 \mathrm{H}), 0.98(\mathrm{t}, J=6.8 \mathrm{~Hz}, 3 \mathrm{H}) ;{ }^{13} \mathrm{C}$ NMR (DMSO$\left.\mathrm{d}_{6}\right): \delta(\mathrm{ppm}) 168.0,166.7,166.3,163.8,161.8,156.5,140.5$, 133.1, 131.9, 131.5, 131.1, 130.0, 123.3, 117.1, 113.4, 62.0, 61.9, 57.3, 57.1, 14.3, 14.1; ${ }^{19} \mathrm{~F}$ NMR (200 MHz, DMSO-d $)$ ) $\delta:-112.0$; MS(ESI): $\mathrm{m} / \mathrm{z}=512\left([\mathrm{M}+\mathrm{H}]^{+}\right), 534\left([\mathrm{M}+\mathrm{Na}]^{+}\right), 550\left([\mathrm{M}+\mathrm{K}]^{+}\right)$. Anal. Calcd for $\mathrm{C}_{22} \mathrm{H}_{20} \mathrm{Cl}_{2} \mathrm{FN}_{3} \mathrm{O}_{4} \mathrm{~S}$ : C, 51.57; H, 3.93; N, 8.20; Found: C, 51.46; H, 3.87; N, 8.15.

Acknowledgements: We are grateful for financial support from the National Natural Science Foundation of China (Grant No. 21602039), the Natural Science Foundation of Guizhou Province (Grant No. Qian Ke He Ji Chu[2017]1066), and the Opening Foundation of the Key Laboratory of Green Pesticide and Agricultural Bioengineering, Ministry of Education, Guizhou University (Grant No. 2018GDGP0102), Science and Technology Program of Guizhou Province (Grant NO. Qian Ke He Ping Tai Ren Cai [2017]5789).

\section{References}

[1] Williams, A. Opportunities for chiral agrochemicals. Pest Manage. Sci. 2015, 46, 3-9.
[2] Massey, M. P. R.; Tandy, M. J. Challenges and frustrations in the separation and analysis of chiral agrochemicals. Chirality 2010, 6, 63-71.

[3] Sekhon, B. S. Chiral pesticides. J. Pestic. Sci. 2009, 34, 1-12.

[4] Liu, H.; Xiong, M. Comparative toxicity of racemic metolachlor and S-metolachlor to Chlorella pyrenoidosa. Aquat. Toxicol. 2009, 93, 100-106.

[5] Zeidler, J.; Baraniak, D.; Ostrowski, T. Bioactive nucleoside analogues possessing selected five-membered azaheterocyclic bases. Eur. J. Med. Chem. 2015, 97, 409-418.

[6] Mfuh, A. M.; Larionov, O. V. Heterocyclic n-oxides - an emerging class of therapeutic agents. Curr. Med. Chem. 2015, 22. 2819-2857.

[7] Dai, H.; Li, G.; Chen, J.; Shi, Y.; Ge, S.; Fan, C.; He, H. Synthesis and biological activities of novel 1,3,4-thiadiazole-containing pyrazole oxime derivatives. Bioorg. Med. Chem. Lett. 2016, 26, 3818-3821.

[8] Haider, S.; Alam, M. S.; Hamid, H. 1,3,4-Thiadiazoles: A potent multi targeted pharmacological scaffold. Eur. J. Med. Chem. 2015, 92, 156-177.

[9] Hu, Y.; Li, C. Y.; Wang, X. M.; Yang, Y. H.; Zhu, H. L. 1,3,4-Thiadiazole: synthesis, reactions, and applications in medicinal, agricultural, and materials chemistry. Chem. Rev. 2014, 114, 5572-610

[10] Yu, P.; Hu, J.; Zhou, T. Y.; Wang, P.; Xu, Y. H. Synthesis, insecticidal evaluation of novel 1,3,4-thiadiazole chrysanthemamide derivatives formed by an $\mathrm{EDCl} / \mathrm{HOBt}$ condensation. J. Chem. Res. 2011, 35, 703-706.

[11] Aly, A. A.; El-Sayed, R. Synthesis and biological activity of new 1,3,4-thiadiazole derivatives. Chem. Pap. 2006, 60, 56-60.

[12] Gan, X.; Hu, D.; Chen, Z.; Wang, Y.; Song, B. Synthesis and antiviral evaluation of novel 1,3,4-oxadiazole/thiadiazole-chalcone conjugates. Bioorg. Med. Chem. Lett. 2017, 27, 4298-4301.

[13] Zhang, K.; Liang, X.; He, M.; Wu, J.; Zhang, Y.; Xue, W.; Jin, L.; Yang, S.; Hu, D. One-pot synthesis of novel chiral $\beta$-amino acid derivatives by enantioselective Mannich reactions catalyzed by squaramide cinchona alkaloids. Molecules. 2013, 18, 6142-6152.

[14] Li, W.; Song, B.; Bhadury, P. S.; Li, L.; Wang, Z.; Zhang, X.; Hu, D.; Chen, Z.; Zhang, Y.; Bai, S. Chiral cinchona alkaloid-derived thiourea catalyst for enantioselective synthesis of novel $\beta$-amino esters by Mannich reaction. Chirality 2012, 24, 223-231.

[15] Song, B. A.; Zhang, H. P.; Wang, H.; Yang, S.; Jin, L. H.; Hu, D. Y.; Pang, L. L.; Xue, W. Synthesis and antiviral activity of novel chiral cyanoacrylate derivatives. J. Agric. Food. Chem. 2005, 53, 7886-7891. 